\title{
Gas adsorbate-induced Au atomic segregation and clustering from $\mathrm{Cu}(\mathrm{Au})$
}

\author{
Lifeng Zhang ${ }^{1}$, Zejian Dong ${ }^{1}$, Shuangbao Wang ${ }^{2}$ and Langli Luo ${ }^{1 *}$
}

\begin{abstract}
Surface compositional and phase segregation in an alloy can change its functionality, especially for applications where surface structure and chemistry play a vital role. For instance, the surface status of alloy catalysts significantly affects their catalytic performance for both heterogeneous and electrochemical processes. Surface segregation is believed to be driven by the difference in surface energy to reduce the total free energy of the alloy. However, the atomistic processes during the segregation process remain elusive, especially for gas molecule-induced segregation, where both structural and chemical reordering may occur. Herein, we achieved in-situ atomic-scale visualization of the surface segregation behaviors of a solid solution $\mathrm{Cu}(\mathrm{Au})$ alloy under the $\mathrm{CO}$ gas by an aberration-corrected environmental transmission electron microscope. $\mathrm{CO}$-induced $\mathrm{Cu}(\mathrm{Au})$ surface ordering structures largely change the surface chemistry of the alloy. Further gas exposure at elevated temperature could facilitate Au atom diffusion through a specific "atomic channel" structure for dealloying and clustering on the surface. The segregated Au nanoparticles show rich phase and morphological dynamics interacting with the alloy surface, where the gas adsorption also plays an important role. These atomic insights provide direct evidence for the surface segregation and dealloying mechanisms of bimetallic alloys, and highlight the role of gas adsorbate in these surface processes.
\end{abstract}

Keywords: surface segregation, clustering, $\mathrm{Cu}(\mathrm{Au})$ alloy, environmental TEM, CO

\section{INTRODUCTION}

The alloy has long been found to go through surface segregation, that is, one constituent is enriched near the surface, which may be accompanied by the surface phase change. The major thermodynamic driving force is the difference in surface energies of alloy constituents-the element with lower surface energy tends to relocate to the alloy surface. Besides, strain in the alloy caused by the difference in atomic radii of alloy constituents could also lead to surface segregation that relaxes the strain level of the alloy system. The above surface segregation behaviors related with "chemical bonding" and "strain" theories have been extensively studied [1-5]. The third type of surface segregation, that is, surface adsorption or surface reaction of gas molecules with one component of the alloy may cause a significant enrichment of that component in the surface of the alloy, receives much less attention [6]. Nevertheless, this type of surface segregation is of great interest for gas-phase heterogeneous catalysis [7] because the metallic surface of catalysts in "working condition" may differ from their "stationary status", both structurally and chemically. And, the dynamic nature of the catalyst's surface plays a decisive role in transforming gas reactants to products. The surface phase and ordering status of bimetallic catalysts are known to be responsible for their superior catalytic properties for a number of catalytic reactions which are more active than their pure counterparts [8-13]. On the other hand, tuning the surface plane, atomic structures, phase, strain and composition of alloy catalysts have created an arsenal of surface engineering strategies to optimize catalytic properties, which are all significantly affected by surface segregation behaviors of alloys.

Surface segregation is often limited to the surface and subsurface atomic layers, hence it is usually investigated under ultra-high vacuum (UHV) conditions with surfacesensitive techniques such as Auger electron spectroscopy (AES), low energy ion scattering spectroscopy (LEIS) and low energy electron diffraction (LEED) $[14,15]$. However, these techniques often lack direct imaging methods with site-specific information and are incompatible with a required reactive gas environment. Scanning tunneling microscopy (STM) has been a major tool to image the

\footnotetext{
${ }^{1}$ Institute of Molecular Plus, Tianjin University, Tianjin 300072, China

${ }^{2}$ School of Physical Science and Technology, Guangxi University, Nanning 530004, China

* Corresponding author (email: luolangli@tju.edu.cn)
} 
surface structure directly, but the information is often limited to the surface and subsurface. Also, the structural dynamics under continuous gas supply is not convenient to obtain due to the mode of "scanning" imaging. Meanwhile, surface segregation may lead to structural and chemical changes beyond subsurface, i.e., a few nanometers deep into the bulk or nanomaterials. Hence, in-situ atomic-scale observations of the whole surface-tobulk region under the dynamic segregation process are essential to decipher underlying atomistic processes and mechanisms. Aberration-corrected environmental transmission electron microscope (AC-ETEM) has emerged as a powerful tool to characterize the dynamic atomic structural change under a gas environment for catalysis research [16-22]. The order-disorder transition on $\mathrm{Cu}(\mathrm{Au})$ surface has been found, and the inter-diffusion of constituent atoms can lead to the formation of dislocations near the alloy surface $[23,24]$. Herein, we demonstrate that surface segregation of $\mathrm{Cu}(\mathrm{Au})$ solid solution alloy could lead to various surface ordering structures and the nucleation of $\mathrm{Au}$ nanoparticles on the surface under the CO exposure. Dynamic structural evolution during the surface segregation and dealloying process has also been captured, which highlights the rich dynamics of surface segregation behaviors under gas exposure.

\section{EXPERIMENTAL SECTION}

\section{Materials preparation}

A model $\mathrm{Cu}-\mathrm{Au}$ solid solution alloy $(\mathrm{Cu}$ and $\mathrm{Au}$ atoms are randomly mixed) thin film ( $\sim 50 \mathrm{~nm}$ thick) with an $\mathrm{Au}$ atomic concentration of $\sim 10 \%$ was prepared by a UHV ebeam evaporation system. The as-prepared $\mathrm{Cu}-\mathrm{Au}$ thin film is single crystal (100) with a thickness of $\sim 50 \mathrm{~nm}$.

\section{AC-ETEM experiments}

The $\mathrm{Cu}-10 \mathrm{at} \% \mathrm{Au}$ thin film was removed from the substrate and mounted on TEM grids through floating in deionized water. A double-tilt Gatan ${ }^{\circledR} 628$ holder was used for heating in a ThermoFisher ${ }^{\circledR}$ Titan ETEM with an objective-lens corrector operated at $300 \mathrm{kV}$, which has a base pressure of $\sim 10^{-8}$ mbar. CO gas was introduced into the ETEM column with a pressure of $0.001 \mathrm{mbar}$ through a leak-valve controlled by ETEM vacuum system. The gas composition was confirmed via a residual gas analyzer (RGA) with sensitivity down to $10^{-11}$ mbar (near the sample area). All experiments were performed at $350^{\circ} \mathrm{C}$.

\section{HRTEM image simulation}

The high-resolution TEM (HRTEM) image simulation of the $\mathrm{Au} \mathrm{NP}$ was carried using the multislicing method, which is integrated into the code "SimulaTEM", as is described in the published paper [25]. The parameters for the image simulation were chosen as follows: accelerating voltage of $300 \mathrm{kV}$ and a spherical aberration coefficient of the objective lens of $2 \mu \mathrm{m}$.

\section{RESULTS AND DISCUSSION}

We used a model single-crystalline $\mathrm{Cu}(\mathrm{Au})$ solid solution alloy $(\mathrm{Cu}$ and $\mathrm{Au}$ atoms are randomly mixed) thin film ( $~ 50 \mathrm{~nm}$ thick) with an Au atomic concentration of $\sim 10 \%$ prepared by a UHV e-beam evaporation system. The asprepared $\mathrm{Cu}-\mathrm{Au}$ thin film was mounted on a heating holder for use in the AC-ETEM. A heating temperature of $\sim 350^{\circ} \mathrm{C}$ and a $\mathrm{CO}$ partial pressure of $\sim 10^{-3}$ mbar were used in all in situ gas-heating experiments. Real-time HRTEM imaging was made from a cross-sectional view of different surface planes, enabling a clear view of both surface and subsurface atoms.

\section{Surface segregation-induced atomic ordering}

The schematics in Fig. 1a illustrate the general behaviors of surface segregation, dealloying and clustering process of the $\mathrm{Cu}-\mathrm{Au}$ alloy. Fig. $\mathrm{lb}$ and $\mathrm{c}$ present typical HRTEM images of pristine $\mathrm{Cu}-\mathrm{Au}(010)$ and (110) surfaces with no observable surface ordering in a vacuum $(<1 \times$ $10^{-8}$ mbar) at $350^{\circ} \mathrm{C}$. Upon CO exposure, surface reordering happens on all surfaces after a staging period ( $\sim 30 \mathrm{~min}$ ), i.e., $\mathrm{Au}$ atoms diffuse to the surface and form a series of ordered structures on the alloy surface. Fig. $1 \mathrm{~d}-\mathrm{i}$ illustrate these surface ordering structures for (010), (110) and (120) surface planes, respectively. In Fig. 1d, the HRTEM image shows a periodicity of brighter and weaker spots in both surface and subsurface atomic layers of (010) surface.

The enlarged image in Fig. 1e shows a good match between the experimental HRTEM image with the corresponding simulated image based on the atomic model of the CuAu-I phase (Fig. S1). Similarly, Fig. 1f-i demonstrate the surface ordering structures, both with a structural motif of $\mathrm{CuAu}_{3}$ phase (Fig. S1) that is enriched with $\mathrm{Au}$ atoms on (110) and (120) surfaces. It is noted that a higher indexed (120) surface can be reconstructed to a double-stepped surface as shown in Fig. 1h, i, and this surface structure can be easily extended to other high-indexed $\mathrm{Cu}(\mathrm{Au})$ surfaces. In addition, lattice distance of $\{100\}$ plane and fast Fourier transform (FFT) image of Fig. $1 \mathrm{~h}$ are also shown in Fig. S2, which can match well with ordered $\mathrm{CuAu}_{3}$ phase. These results collectively reveal that the disorder-to-order transition is 

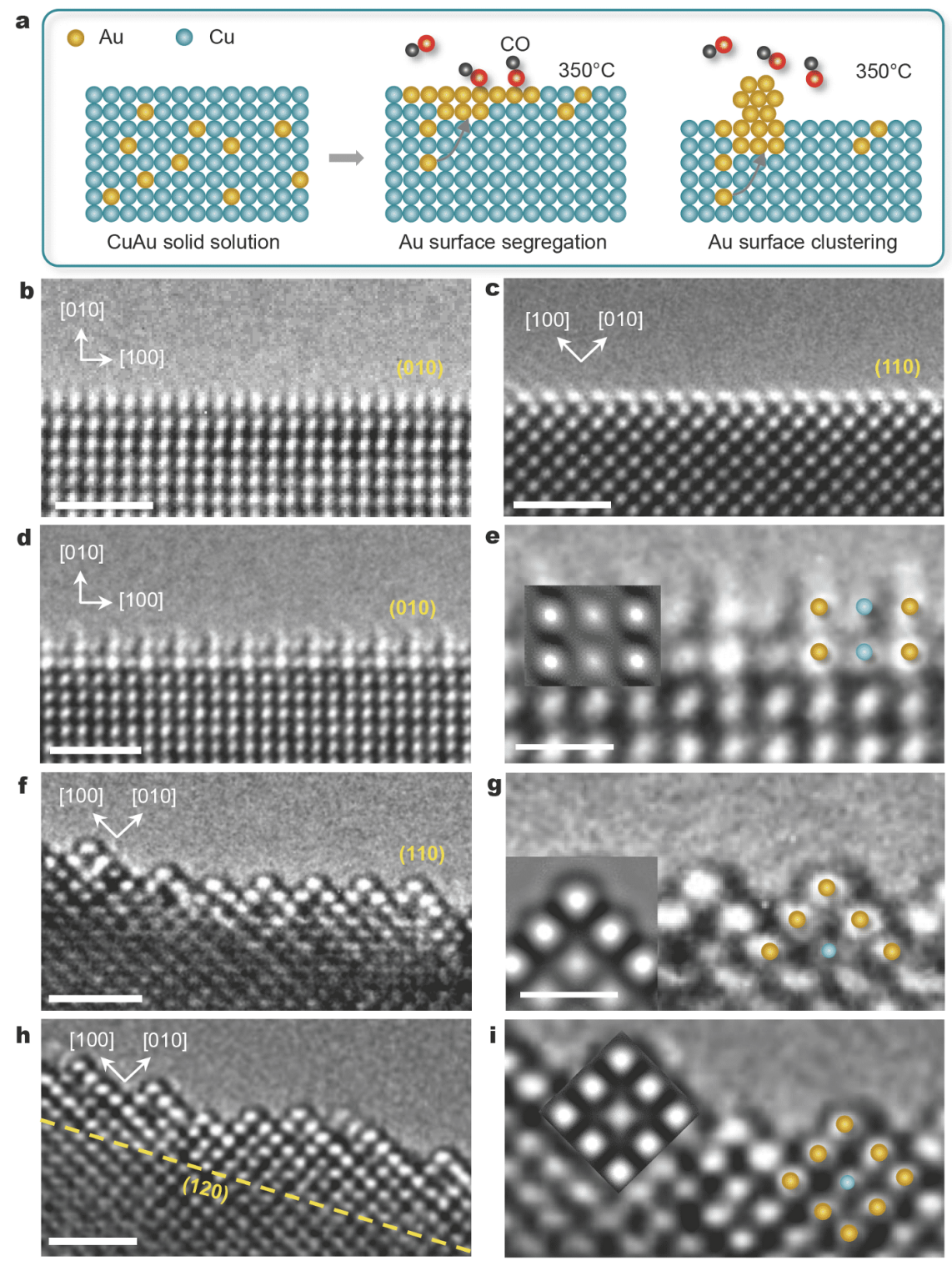

Figure 1 Atomic-scale surface segregation of $\mathrm{Cu}$-Au alloy. (a) Schematics of the Au surface segregation and clustering process on the $\mathrm{Cu}(\mathrm{Au})$ alloy upon $\mathrm{CO}$ exposure. (b, c) Typical HRTEM images of pristine $\mathrm{Cu}(\mathrm{Au})(010)$ and (110) surfaces with no observable surface segregation in a vacuum. (di) HRTEM images of the surface ordering structures for (010), (110) and (120) surface planes and enlarged images are presenting good matches with simulated images based on atomic models of different ordering phases. Scale bars are $1 \mathrm{~nm}$ (b, c, d, f, h), and $5 \AA$ (e, g, i), respectively.

a general behavior for a solid-solution binary alloy, and the Au concentration can be readily enriched to 75 at $\%$ of $\mathrm{CuAu}_{3}$ ordered phase on the $\mathrm{Cu}-\mathrm{Au}$ surface.

The above $\mathrm{Au}$ atom segregation-induced ordering at the $\mathrm{Cu}(\mathrm{Au})$ surface can usually reach a thermodynamic equilibrium when the total surface energy and strain energy are minimized by these ordering structures. Surprisingly, in the presence of $\mathrm{CO}$, we observed further surface segregation-induced dynamic structural changes near the surface with elongated exposure time. After
60 min of staging under $\mathrm{CO}$ gas at $350^{\circ} \mathrm{C}$, some stepped alloy surfaces went through more complicated compositional segregation accompanied by vigorous atomic rearrangement. In Fig. 2a, multiple atomic steps are present on the $\mathrm{Cu}(\mathrm{Au})$ surface, which consists of (010) surfaces and some higher-indexed sloped surfaces connecting them (white arrows). These surface steps do not adopt surface reconstructed structures observed in Fig. 1, but show unique atomic structures (bottom-left insets) featuring a gradual increase of the lattice distance between 


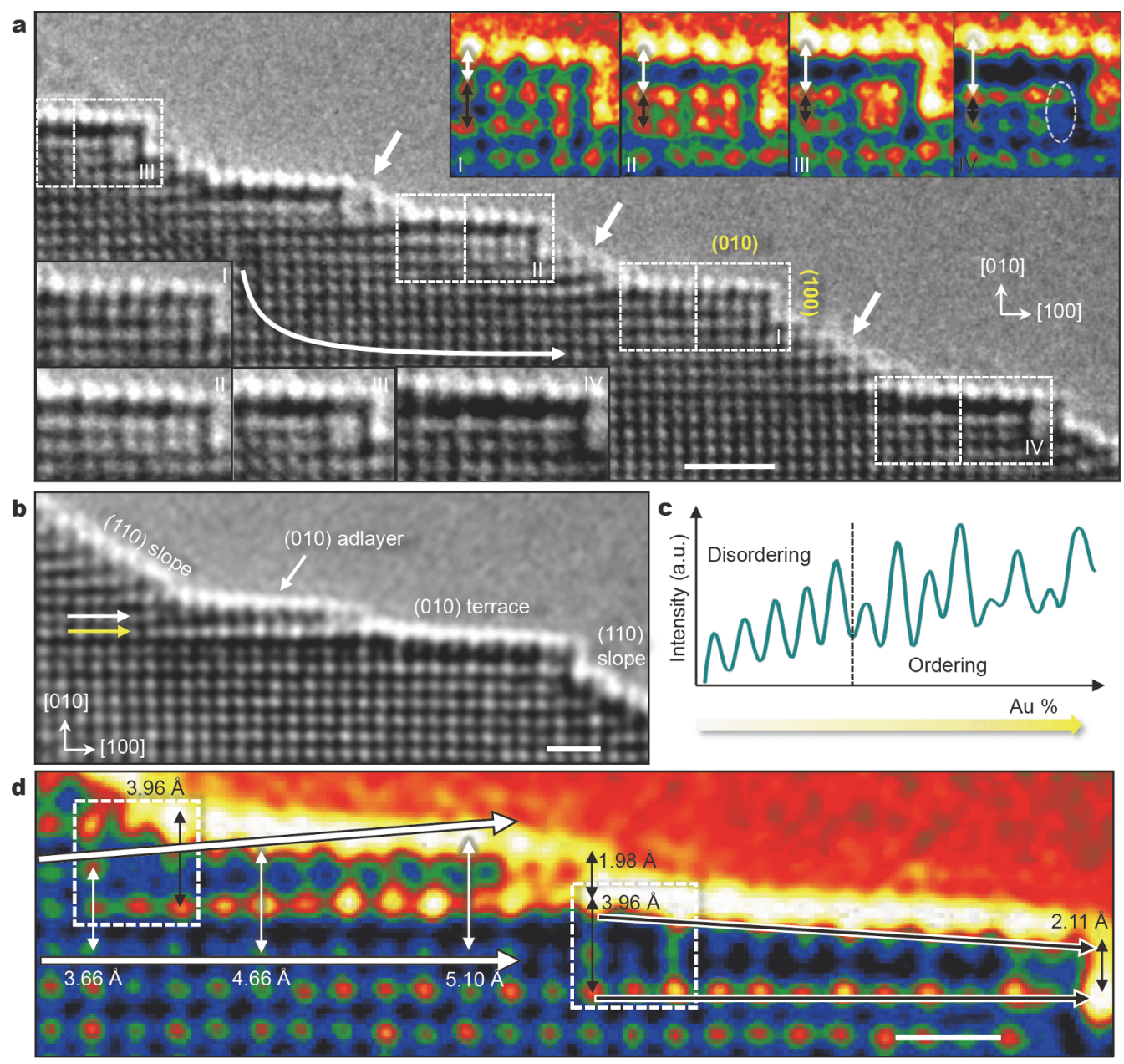

Figure 2 "Atomic channeling" facilitates surface segregation in CO. (a) HRTEM image shows multiple surface steps with different stages (I to IV, white-dashed line boxes) of Au surface segregation process defined by the lattice distance between surface and subsurface layer (insets). (b) The typical HRTEM image presents the atom rearrangement near surface step during the segregation process, which may enable a possible "atomic channeling" path for diffusion. (c) Intensity profile shows the disorder-to-order transition of $\mathrm{Cu}(\mathrm{Au}$ ) lattice along the direction from bulk to surface (yellow arrow in (b)). (d) Detailed structural analysis of the "atomic channeling" shows an increase of lattice distance from 3.66 to $5.10 \AA$ for the upper terrace and a decrease of lattice distance from 3.96 to $2.11 \AA$ for the upper terrace. The scale bars are $1 \mathrm{~nm}$ (a), and $5 \AA$ (b, d), respectively.

the surface and subsurface. Depending on the degree of lattice expansion, stages I, II, III, and IV are labeled for different surface steps, although they are captured at the same time. The up-right insets clearly illustrate this gradual change of the step-edge areas, where the lattice distance between the surface and subsurface atom layers keeps increasing from 1.98 to $3.31 \AA$ (white double-arrows) while the lattice distance between the subsurface layer and the $3^{\text {rd }}$ sublayer keeps decreasing from 1.98 to $1.75 \AA$ (black double-arrows). This trend of lattice expansion between the surface and subsurface planes has also been found on (100) surface plane (labeled by yellow indexes), which means it is isotropic for the cubic structure. As shown in the later stage (IV) of this surface change, one or more atom columns are missing between the surface and subsurface layer as indicated by the whitedashed ellipse.

As shown in Fig. 2b and Movie S1, a (010) terrace with a half adlayer (white arrow) becomes the dominating structure on these stepped alloy surfaces. This "terraceadlayer" structure features a (010) terrace separated by two (110) slope surfaces, and a half adlayer is covered on the (010) terrace extending from upper (110) surface. One subsurface (010) atomic plane (white arrow) beneath the half adlayer extends from the bulk alloy to the surface and merges with the (010) adlayer. This structural relaxation leads to a disorder-to-order transition in the atomic plane beneath the adlayer (yellow arrow). The intensity profile 
of atoms in this plane from bulk to the surface is drawn in Fig. 2c, which shows a clear disorder-to-order transition along this atomic plane. This is also accompanied by the increase of Au concentration from the bulk to the surface but with a deeper penetration to the bulk comparing those surface ordering structures in Fig. 1. In addition, atomic layer shown by the white arrow in Fig. $2 \mathrm{~b}$ disappears gradually between the surface and subsurface atom layers in (010) adlayer (Fig. S3), while the intermediate region between them is still occupied by some atomic columns. This observation explains the large expansion from 1.98 to $3.31 \AA$ (stages I to IV).

Detailed analysis in this region reveals a possible $\mathrm{Au}$ diffusion channel from bulk to the surface enabled by this "terrace-adlayer" structural feature. As shown in Fig. 2d, two big white arrows indicate the atomic layers above and under the disorder-to-order transition layer, which go through a large expansion from bulk to the surface. The $d_{(010)}$ increases from $3.66 \AA(\mathrm{Cu}-10$ at $\% \mathrm{Au})$ to $5.10 \AA$ near the step-edge and this expanded lattice should largely promote $\mathrm{Au}$ diffusion from bulk to the surface. Two white-dashed boxes show a structural unit with its structure and dimension identical with that of $\mathrm{Au}_{3} \mathrm{Cu}$ $(\sim 3.96 \AA)$. These two units are right on the connection between the (110) slope and the (010) adlayer, which initiates a surface structure transition from a lattice distance of two (020) layers $(\sim 3.96 \AA)$ to around one (020) layer $(\sim 2.11 \AA)$ as shown by two black arrows. This two-to-one atom layer transition leads to the diminishing of atoms, and thus frees the space above the "disorder-to-order" layer. These observations illustrate complicated segregation-induced atomic restructuring and also chemical modifications at the surface and near-surface regions, especially at the stepped alloy surface. These changes are primarily induced by the tendency of $\mathrm{Au}$ segregation driven by strong CO-metal interaction, especially for those low-coordinated atoms near step edges. $\mathrm{Au}$ and $\mathrm{Cu}$ atoms have been reported to have a good affinity to $\mathrm{CO}$ molecules and form $\mathrm{Au}-\mathrm{CO}$ or $\mathrm{Cu}-\mathrm{CO}$ species under $\mathrm{CO}$ gas at elevated temperature [26-28]. These behaviors are absent under thermal-only conditions, which highlights the effect of gas adsorbate on surface segregation. It is also noted that the surface diffusion also promotes this segregation behavior kinetically, where the enhanced surface diffusion caused by large surface area and the presence of surface steps can facilitate the exchange of $\mathrm{Au}$ and $\mathrm{Cu}$ atoms on the alloy surface.

\section{Nucleation of segregated Au atoms}

Dealloying and clustering at the surface often require large chemical driving force beyond the traditional regime of surface segregation. Here, we find elongated $\mathrm{CO}$ exposure could induce such a phenomenon, that is, $\mathrm{Au}$ segregation leads to the nucleation of Au nanoclusters on the alloy surface, as shown below. In Fig. 3, two different modes of Au nucleation have been observed. The first one proceeds through gradual segregation of ordered $\mathrm{Au}$ clusters at step-edge sites with an epitaxial interface. Time-resolved HRTEM images recorded this process in Fig. 3a. Initiated at step-edges indicated by two white arrows, $\mathrm{Au}$ atom clusters increase their dimensions laterally with time and eventually reside on the terraces created by the segregated atoms. Au atoms pre-segregated to the high-indexed alloy surface (Fig. 1d-i) can be the source of $\mathrm{Au}$ atoms to form this type of $\mathrm{Au}$ clusters through short-length diffusion. The surface steps are covered with $\mathrm{Au}$ clusters, which lowers the total free energy in this model. Hence, these Au clusters are stabilized on these steps rather than merge into bigger nanoparticles. The second mode also initiates from the stepedge but the nucleus does not follow the epitaxial relationship with the substrate, as shown in time-resolved HRTEM images in Fig. $3 \mathrm{~b}$ and Movie S2. The Au atoms aggregate on the step-edge and evolve from a disordered structure to a nanoparticle as shown in Fig. 3c, which possesses a well-defined face-center-cubic (FCC) structure with well-faceted (111) and (010) planes (Fig. S4). It is noted that these two modes can trace back to the specific "terrace-adlayer" structure (indicated by white dashed-line boxes described in Fig. 2), which disappears after the surface clustering as seen in the image of $76 \mathrm{~s}$ in Fig. $3 \mathrm{a}$ and the image of $58 \mathrm{~s}$ in Fig. 3b. This observation indicates the dealloying and clustering of $\mathrm{Au}$ atoms are largely facilitated through the unique "atomic channeling" process.

Through surface segregation and clustering, Au atoms either form supported nanoparticles (Fig. 3d) or coherent interface (Fig. 3f) with alloy substrate with an epitaxial relationship. As shown in Fig. 3d, a gold nanoparticle with a size of $\sim 4 \mathrm{~nm}$ is supported on the (110) surface and corresponding FFT pattern shows a good epitaxial relationship between them. It is noted that considerable strain exists in the particle-substrate interface as shown in the strain analysis from the HRTEM image in Fig. 3e. As shown in Fig. 3f, a coherent interface is shown in the HRTEM image and the corresponding FFT pattern, but one transition region (TR) with "band-like" structure can be observed between $\mathrm{Au}$ and the alloy substrate. Detailed analysis shows no misfit dislocation was formed at the interface between the $\mathrm{CuAu}$ substrate and TR while sev- 

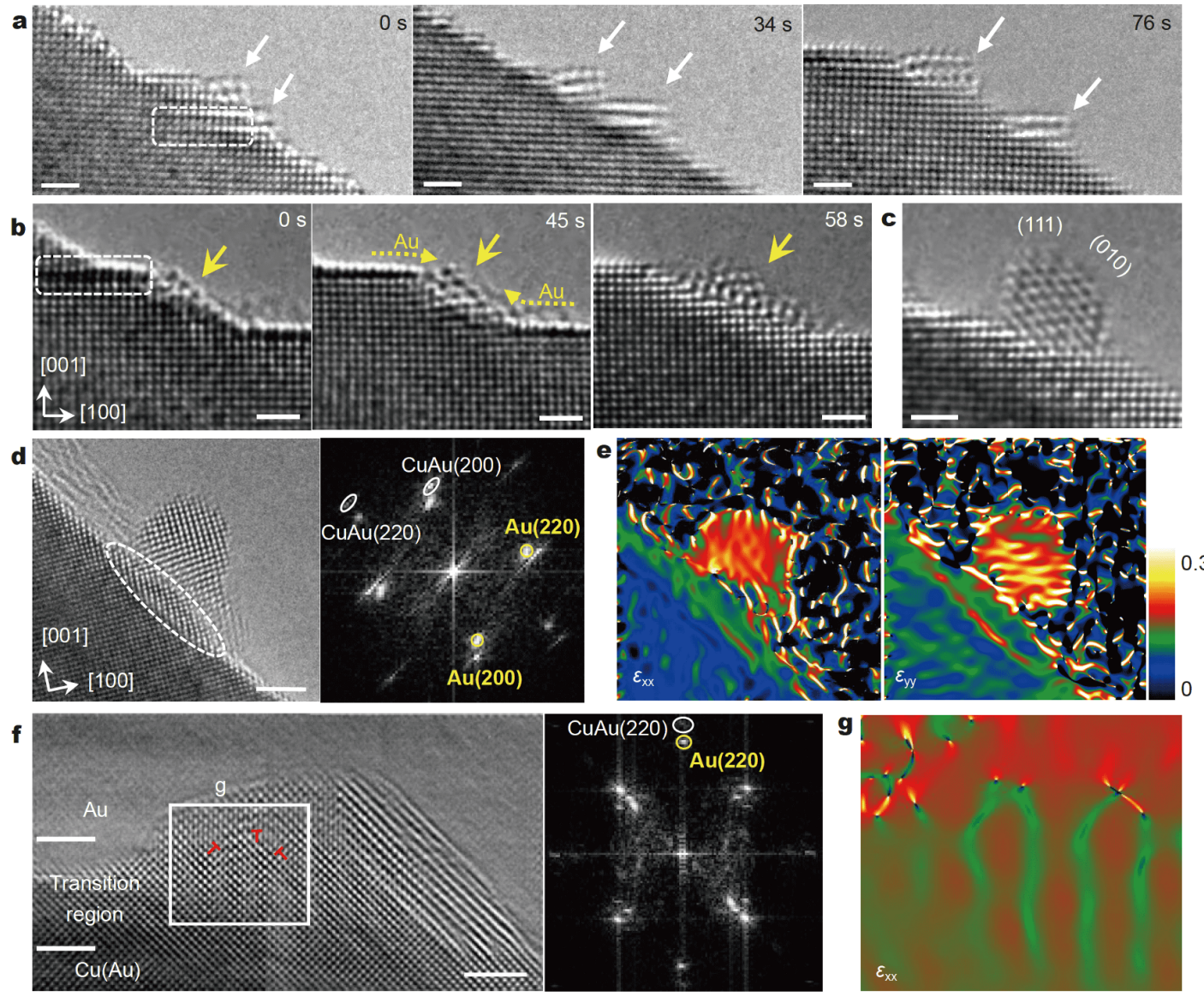

Figure 3 In situ observation of Au segregation and clustering. (a, b) Time-resolved HRTEM images show two different nucleation modes (epitaxy $v$ s. particle) of Au clustering at step-edges. (c) Typical HRTEM image of a segregated Au nanoparticle stabilized at the step-edge of Cu-Au substrate. (d) HRTEM image and corresponding FFT pattern show an epitaxial relationship between the segregated Au nanoparticle and alloy substrate. (e) The corresponding strain analysis shows a strained interfacial area (white-dashed line ellipse in (d)). (f) HRTEM image and corresponding FFT pattern show segregated Au atoms "wet" on the surface, and a TR forms between an intimate epitaxial interface and the alloy substrate. (g) Strain analysis of the "inter-band" atomic structure shown by rectangular zone in (f). All the scale bars are $1 \mathrm{~nm}$.

eral misfit dislocations were formed at the $\mathrm{Au} / \mathrm{TR}$ interface as shown by the strain analysis in Fig. $3 \mathrm{~g}$ and Fig. S5. Since the lattice of "band-like" TR is acquired under different defocus values (Fig. S5), we deduce that "bandlike" structure in TR was formed by atomic-scale bending. Diffusion of $\mathrm{Au}$ atoms from alloy to the surface results in an $\mathrm{Au}$ concentration increase in TR while maintaining a coherent interface between them. Atomicscale bending can be seen as a superstructure that releases the lattice mismatch between alloy substrate and TR.

\section{Stability of Au clusters on alloy substrate}

After $\mathrm{Au}$ atoms have segregated to the alloy surface in the form of supported nanoclusters, they show rich dynamic interaction behaviors with the substrate. We find surface steps play a critical role in stabilizing these supported $\mathrm{Au}$ clusters. Time-resolved HRTEM images in Fig. 4a captured from Movies S3 and S4 present how an Au nanocluster near a step-edge changes its crystallographic orientation with substrate not only by rotating but also by reshaping its morphology to transform between two metastable states. It should be noted that we can clearly see the lattice of Au cluster only if the speed of morphological change is slower than the temporal resolution of the camera $(\sim 0.5 \mathrm{~s})$, and the observed phase can be considered as a metastable state. In the image of $0 \mathrm{~s}$, the $\mathrm{Au}$ cluster is located on a step with 2 layers of (020) lattice, and the interface between Au cluster and substrate has a semi-coherent relation that the Au (111) plane is extended from (020) plane of the alloy with a small tilt as indicated by the yellow dashed line. Obviously, this interface is in a metastable status because of the large dif- 

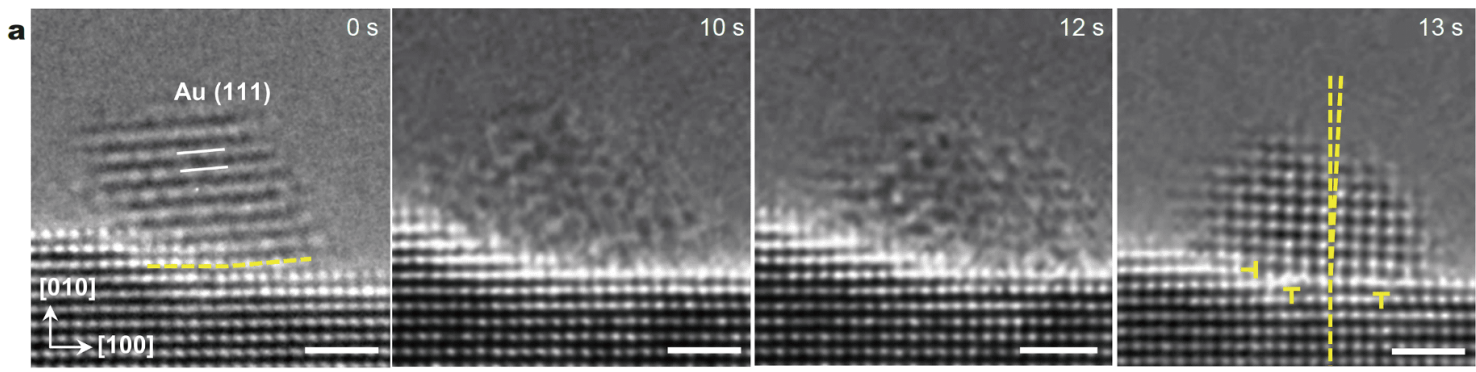

b
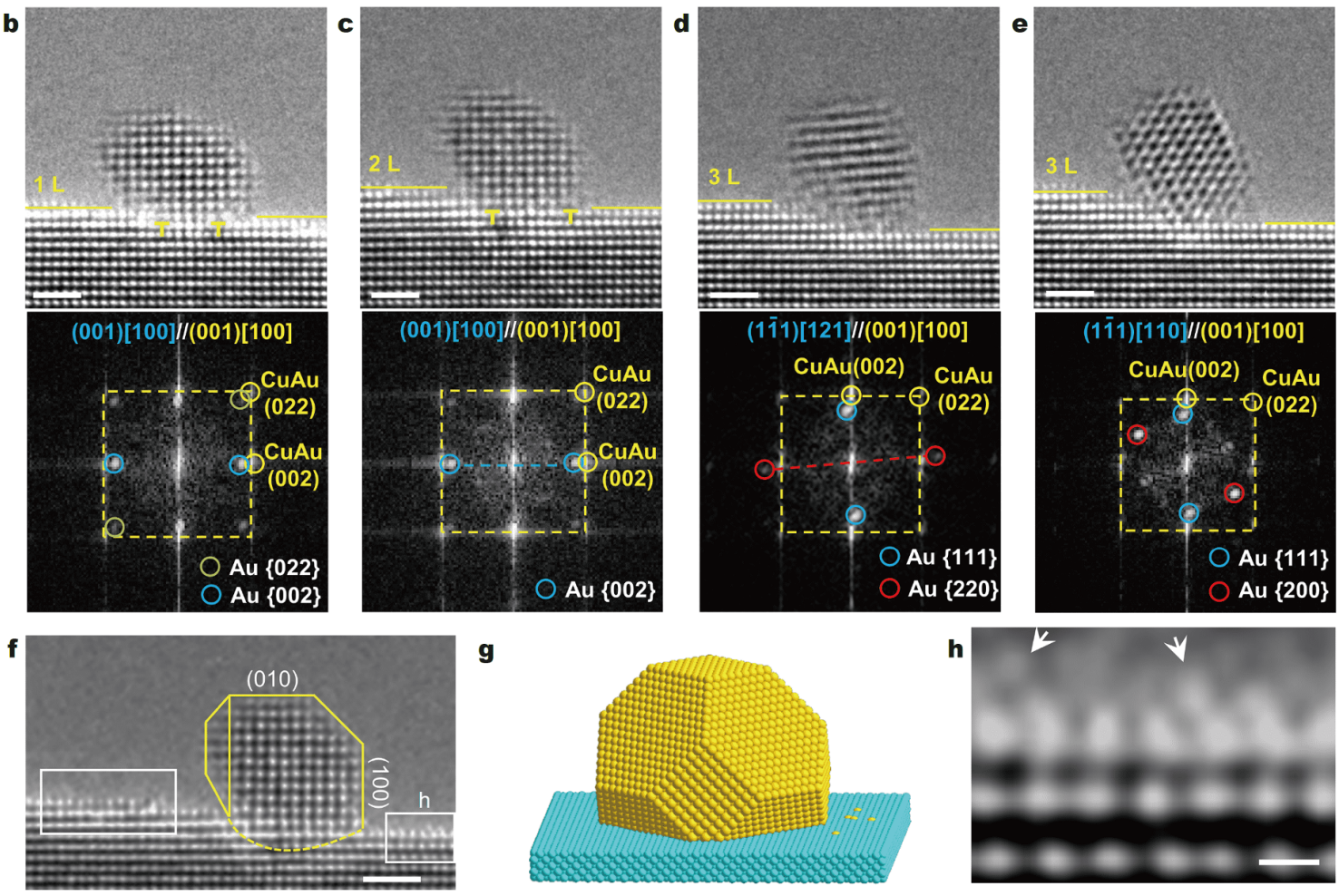

g
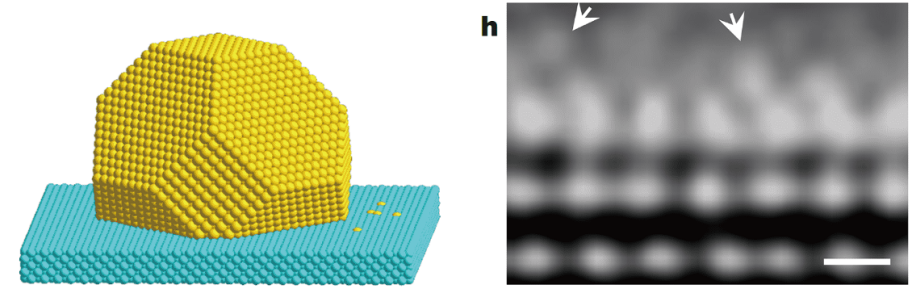

Figure 4 Dynamic balance of segregated Au nanoparticles induced by CO adsorption. (a) Time-resolved HRTEM images show the rotation and morphological change of an Au nanoparticle near a step-edge. (b-e) HRTEM images and the corresponding FFT patterns demonstrating various Au cluster-substrate orientations during the dynamic movement of cluster Au. (f, g) HRTEM image and the schematic of the Au nanoparticle with equilibrium shape using the Wulff construction, respectively. (h) Enlarged image of the rectangular zone in (f) shows possible "single" Au atom on the surface. The scale bar in (h) is $2 \AA$, all other scale bars are $1 \mathrm{~nm}$.

ference between the lattice distance of $\mathrm{Au}(111) \sim 2.35 \AA$ and $\mathrm{CuAu}_{3}(020) \sim 2.0 \AA$. Hence, it quickly changes its shape through atomic rearrangement in the nanocluster and reaches a stable status after $13 \mathrm{~s}$. The final clustersubstrate orientation is close to a cube-on-cube epitaxial but with a small tilt (indicated by two yellow dashed lines), and three lattice-mismatch dislocations are found on their interface.

We captured various cluster-substrate geometries during the dynamic movement of Au cluster on the substrate as shown in Fig. 4b-e and Fig. S6. Two metrics can be seen from the corresponding FFT patterns below the
HRTEM images, i.e., the number of atomic layers of the step and the cluster-substrate orientation. In Fig. $4 b, c$, the Au clusters are both in a cube-on-cube epitaxial relationship with the substrate, i.e., $(001)[100]_{\mathrm{Au}} / /$ (001)[100 $]_{\text {Sub }}$ with 1 layer and 2 layers of (020) atomic plane, respectively. In Fig. $4 \mathrm{~d}$, the Au cluster shows a small tilt $\left(\sim 4^{\circ}\right)$ between its $(220)_{\text {Au }}$ plane and (100) plane of the substrate with a three-layer step. In Fig. 4e, the cluster-substrate orientation is $(1 \overline{1} 1)[110]_{\mathrm{Au}} / /(001)[100]_{\mathrm{Sub}}$, and it also resides on a three-layer step site. According to the Wulff construction theory, the Au cluster can reach an equilibrium shape with all low-indexed $\{100\}$ and 
$\{111\}$ surfaces exposed as seen in Fig. 4f, g and Fig. S7. Au particles can extend into the $\mathrm{Cu}(\mathrm{Au})$ substrate, as shown by the dotted arc in Fig. $4 \mathrm{f}$ and Fig. S8, where the interfacial strain is mediated by misfit dislocations. From the HRTEM image of an enlarged area (white box in Fig. 4f), we could also spot traces of separated Au clusters down to single atom as indicated by white arrows in Fig. $4 \mathrm{~h}$, which indicates a mass transport may be ongoing between the nanoparticle and alloy substrate.

The above observation of dynamic interaction of supported $\mathrm{Au}$ nanoparticles with substrate indicates that the particle-substrate orientation is not fixed and can be tuned by a small perturbation caused by local thermal and/or chemical fluctuation. Meanwhile, the Au nanoparticle can go through atom restructuring to response to this local variation and reach several metastable states. Therefore, we provide a semi-quantitative model to describe this gas-adsorbate involved structural dynamics of supported clusters. Morphology of Au nanoparticles and structural relation with the substrate are governed by the total free energy contributed by surface energy of the $\mathrm{Au}$ nanoparticle, particle-substrate interfacial energy and adsorption energy of $\mathrm{CO}$ gas molecules. We focus on two of the most stable states during the dynamical changes of cluster-substrate relationship as shown in Fig. $5 \mathrm{a}$, b, both of which last for a few seconds in our video recording. It is seen that the Au nanoparticle in Fig. $5 \mathrm{a}$ is close to the equilibrium shape, and two misfit dislocations can be observed, while one misfit dislocation exists in Fig. 5b. The interfacial area of $\mathrm{Au}$ nanoparticle and $\mathrm{Cu}(\mathrm{Au})$ substrate decreased gradually from Fig. $5 \mathrm{a}$ to $\mathrm{b}$. This "dewetting" process is clearly shown in the Movie S3. Thus, we assume that the out-most $\mathrm{Au}$ atoms on $\{111\}$ facets of $\mathrm{Au}$ nanoparticle are detached layer by layer in this "dewetting" process, as shown by Au atoms (tan color) and the black arrows in Fig. 5c.

In order to quantify the systematic energy, Fig. $5 c-e$ show the schematic to calculate the change of surface energy of Au nanoparticle (details in the Supplementary information). We use a generalized Wulf-Kaishew theorem [29] to roughly quantify the supported Au nanoparticle under CO gas environment as follows. The total free energy $(E)$ is composed of the surface energy of $\mathrm{Au}$ nanoparticles $(\sigma), \mathrm{Au} / \mathrm{Cu}$-Au alloy interfacial energy $\left(E_{N}\right)$ and the adsorption energy of $\mathrm{CO}$ molecules $\left(E_{\mathrm{a}}\right)$, shown in Equation (1). And we use the following Equations (2) and (3) to acquire the total energy change per $0.05 \mathrm{~nm}$ $\left(\Delta E^{\prime}\right)$ with the interfacial width.

$$
\begin{aligned}
& \begin{aligned}
E & =\sigma+E_{N}+E_{\mathrm{a}}=\sigma+\left(E_{\mathrm{d}}+E_{\varepsilon}\right) S+E_{\mathrm{a}} \\
& =\sum_{i \neq \mathrm{AC}} \gamma_{i} S_{i}+\left(\gamma_{\mathrm{AC}}-\gamma_{\mathrm{C}}\right) S_{\mathrm{AC}}+\left(\frac{G_{\mathrm{A}} G_{\mathrm{C}}(f-\varepsilon) b}{\pi\left(G_{\mathrm{A}}+G_{\mathrm{C}}\right)(1-v)}\left(\ln \frac{R}{b}+1\right)+\frac{2 G_{\mathrm{A}}(1+v)}{1-v} h \varepsilon^{2}\right) S_{\mathrm{AC}}+\sum_{j \neq \mathrm{AC}} \gamma_{j}^{\prime} S_{j},
\end{aligned} \\
& \begin{aligned}
\Delta E & =E-E_{0}=\Delta \sigma+\Delta E_{N}+\Delta E_{\mathrm{a}} \\
& =\left[\gamma_{100}\left(S_{100}^{1}-S_{100}^{0}\right)+\gamma_{111}\left(S_{111}^{1}-S_{111}^{0}\right)\right]_{\mathrm{A}}-\gamma_{100} \Delta S_{\mathrm{C}}+\left[\frac{G_{\mathrm{A}} G_{\mathrm{C}}(f-\varepsilon) b}{\pi\left(G_{\mathrm{A}}+G_{\mathrm{C}}\right)(1-v)}\left(\ln \frac{R}{b}+1\right)+\frac{2 G_{\mathrm{A}}(1+v)}{1-v} h \varepsilon^{2}\right] \Delta S_{\mathrm{C}},
\end{aligned} \\
& \Delta E^{\prime}=E_{x_{0}+0.05}-E_{x_{0}}=\left(E_{x_{0}+0.05}-E_{0}\right)-\left(E_{x_{0}}-E_{0}\right)=\Delta E_{x_{0}+0.05}-\Delta E_{x_{0},}
\end{aligned}
$$

where $\sigma$, for a crystal having $i$ facets of area $S_{i}$ characterized by the surface energy $\gamma_{i} S_{i} \cdot \gamma_{\mathrm{AC}}$ is the interfacial energy density and $S_{\mathrm{AC}}$ is the contact area. $f=\left(a_{\mathrm{A}}-a_{\mathrm{C}}\right) / a_{\mathrm{C}}$ is the epitaxial misfit between $\mathrm{Au}$ (lattice parameter $a_{\mathrm{A}}$ ) and $\mathrm{Cu}$ substrate (lattice parameter $a_{\mathrm{C}}$ ), which was shared by dislocations and strains. That is, $f$ can be divided into the elastic strain $\varepsilon$ and $\delta$, misfit accommodation by dislocations. It means that there are $N$ interfacial dislocations at the interface to release the misfit, and $\delta=N|\boldsymbol{b}| / L$, in which $\boldsymbol{b}$ is Burger's vector component of misfit dislocations. So interfacial width $L$ can be expressed to be $N d+x(0<x<$ $d$. $d$ is the separation of parallel misfit dislocations), and $S_{\mathrm{AC}}$ can be roughly expressed to be $L^{2} . R$ is the distance to the outmost boundary of the dislocation's strain field. If
$2 d$ is less than the height of Au particle $h$, then $R \approx d . G_{\mathrm{A}}$, $G_{\mathrm{C}}$ and $v$ are the shearing modulus and Poisson ratio of $\mathrm{Au}$ and $\mathrm{Cu}$, respectively. In addition, we provided the calculation process for systematic energy in Section "Total energy calculation" of Supplementary information and Figs S9, S10.

As shown in Fig. 5f, we assume that the initial interfacial width $L$ of $\mathrm{Au}$ particle and $\mathrm{Cu}(\mathrm{Au})$ substrate is about $3 d$. Since the lattices between $\mathrm{Au}$ and $\mathrm{Cu}$ substrate tend to be ordered in middle of adjacent two misfit dislocations $(0<x<d)$, there is one minimal value for the strain energy $E_{N}$, meaning that $\Delta E^{\prime}$ increases first, then decreases in the range of $0<x<d$. Positions of misfit dislocation illustrated by " $N=1,2,3$ and 4 " are the 

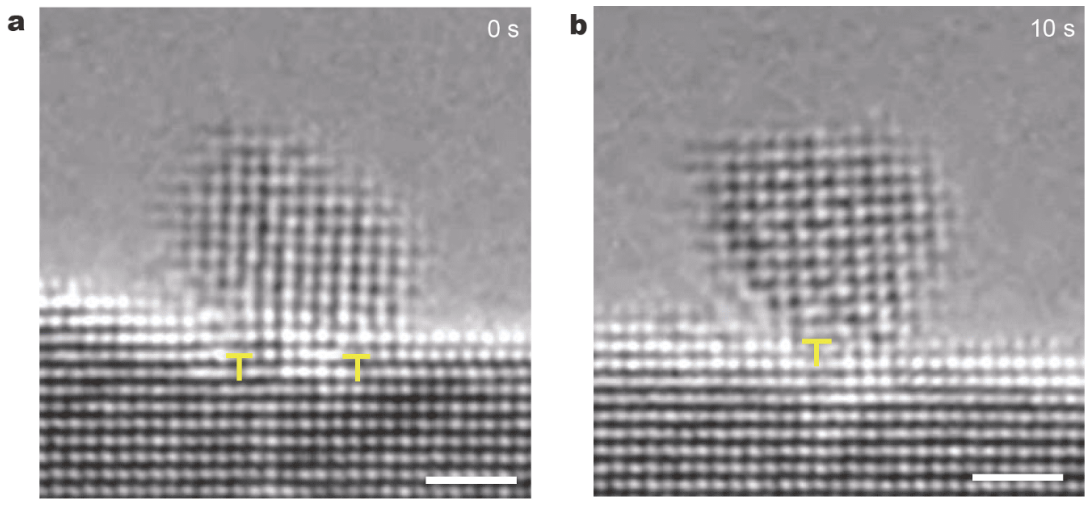

C

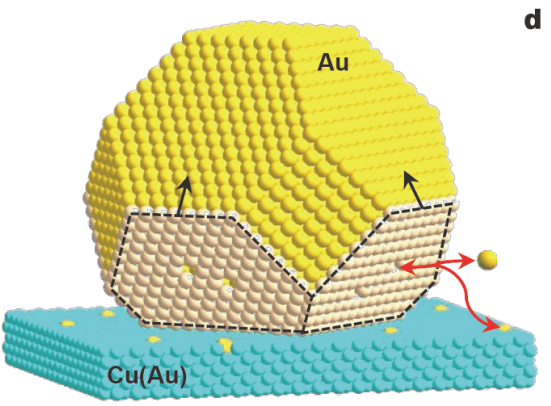

d

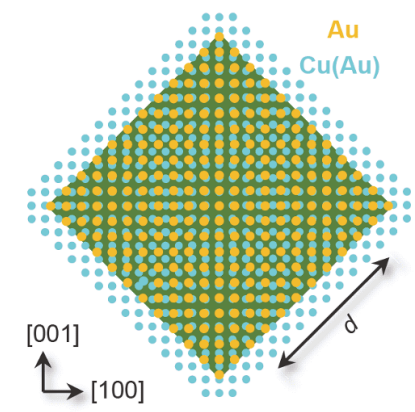

e
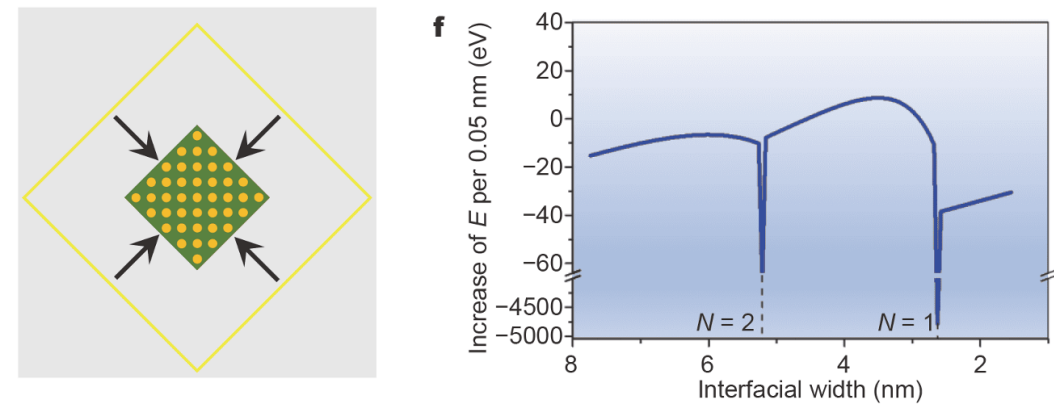

Figure 5 Thermodynamic characterization of the "dewetting" Au nanoparticles. (a, b) Time-resolved HRTEM images showing the dewetting process of Au nanoparticle on the $\mathrm{Cu}(\mathrm{Au})$ substrate. Both scale bars are $1 \mathrm{~nm}$. (c) Schematic of the Au nanoparticle with equilibrium shape. We assumed that out-most $\mathrm{Au}$ atoms on $\{111\}$ surface of Au nanoparticle were escaped layer by layer, as shown by tan Au atoms and the black arrows. (d, e) Schematic model of "atom peeling" process describing facet change (d) and the interfacial area change (e) of Au nanoparticle and $\mathrm{Cu}(\mathrm{Au})$ substrate. (f) $\mathrm{Change}$ of total energy $E$ per $0.05 \mathrm{~nm}$ with the interfacial width.

abrupt drop in systematic energy, because one misfit dislocation disappears. This causes the instability of supported nanoparticles due to the structural relaxation. Besides, compared with the change of systematic energy, disturb of temperature change and $\mathrm{CO}$ flow may be the major factor causing the morphology and orientation instability of Au nanoparticles, when the interfacial width $L$ is less than the separation of misfit dislocation $d$. Above analysis shows the origin of the interfacial structure instability between small nanoparticle and the support, which are encountered in real gas environment at elevated temperature.

Coalescence of Au clusters can also happen on the alloy surface featured by a move-and-merge mechanism. In Fig. 6a, time-resolved HRTEM images illustrate four Au clusters $(2-3 \mathrm{~nm})$ gradually move and coalesce together one by one and finally form a nanoparticle $(5-6 \mathrm{~nm})$ as tracked by yellow-dashed circles. The final nanoparticle has multigrain with clear grain boundary as shown in Fig. 6b $(0 \mathrm{~s})$, and the grain boundary can disappear during continuous annealing and gas exposure after $380 \mathrm{~s}$. This similar coalescence behavior is also found in liquid with the help of capping agents, demonstrating how five-fold symmetry Au nanoparticle is formed [30]. Our observations on further coalescence events can lead to similar results as shown in Fig. $6 c$, d. It is also noted 


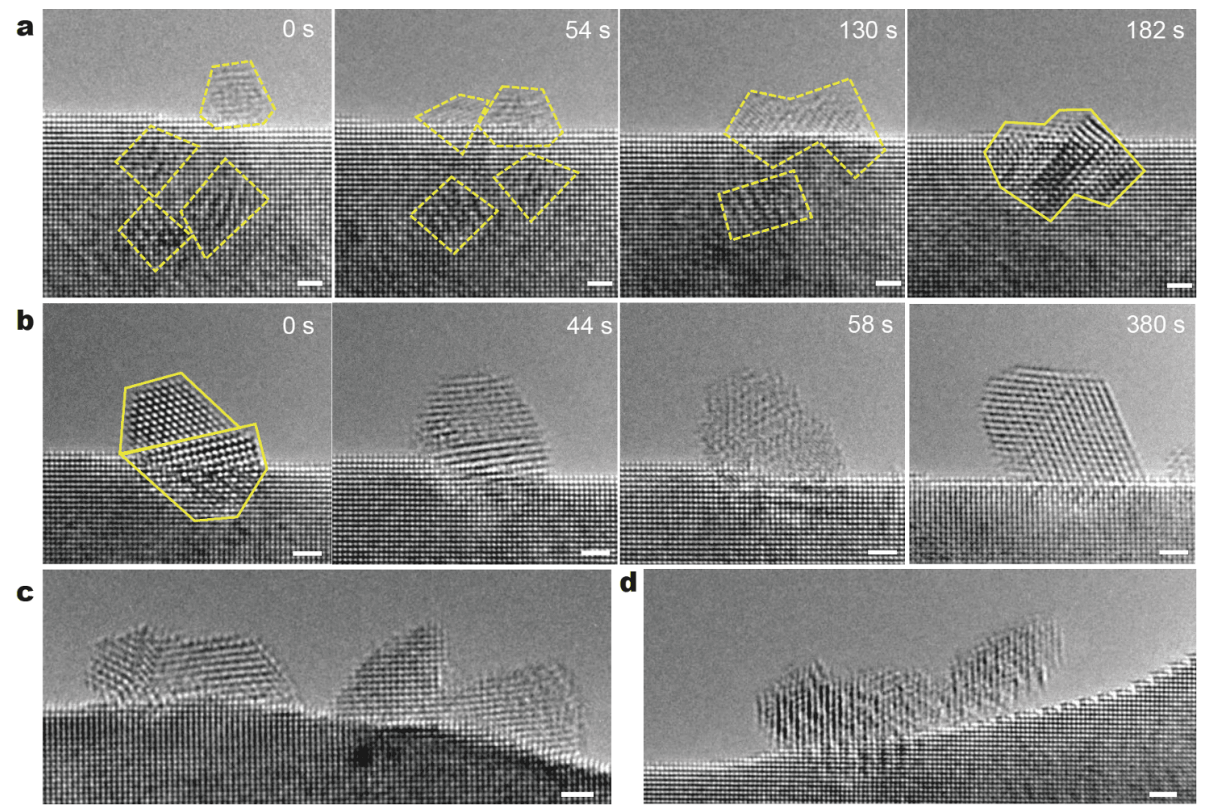

Figure 6 Coalescence of Au nanoparticles on the substrate. (a) Time-resolved HRTEM images show the coalesce process of four Au nanoparticles into a stable large Au nanoparticle. (b) Time-resolved HRTEM images show the process of diminishing of grain boundaries of a coalesced Au nanoparticle. (c, d) HRTEM images show the similar coalescence process of larger Au nanoparticles. All the scale bars represent $1 \mathrm{~nm}$.

that the movement of small Au clusters is largely limited on the alloy surface, i.e., they can only migrate on the surface, in contrast with free-movement in liquid media; however, they share similar mechanistic features as shown above.

\section{CONCLUSIONS}

We investigate atomic-scale $\mathrm{Au}$ segregation-induced dynamic behaviors on the surface of a solid solution $\mathrm{Cu}-$ 10 at $\% \mathrm{Au}$ alloy under $\mathrm{CO}$ gas exposure at $350^{\circ} \mathrm{C}$ by $\mathrm{AC}-$ ETEM. We directly visualize a series of real-time observations induced by Au segregation including surface ordering, dealloying and nucleation of Au clusters and dynamic evolution of Au clusters to nanoparticles as well as their stability on the alloy surface. Remarkably, we find a unique atom channeling effect on the alloy (010) surface, which facilitates the Au atomic transportation from bulk to the surface during segregation. We also find various types of particle-substrate orientation relationships and rationalize it with a quantitative model considering surface energy, interface energy and gas adsorption energy, to explain how they transform among different geometries. These results provide atomic insights to the fundamental understanding of surface segregation and dealloying and can be extended to applications involving such alloy system such as phase evolution of supported nanoparticle catalysts.
Received 2 August 2020; accepted 22 September 2020; published online 14 December 2020

1 McDavid JM, Fain Jr. SC. Segregation at Cu-Au alloy surfaces. Surf Sci, 1975, 52: 161-173

2 Nelson GC. Summary abstract: Surface composition of $\mathrm{Cu} / \mathrm{Au}$ alloys. J Vac Sci Technol A, 1983, 1: 1037-1038

3 Buck TM, Wheatley GH, Marchut L. Order-disorder and segregation behavior at the $\mathrm{Cu}_{3} \mathrm{Au}$ (001) surface. Phys Rev Lett, 1983, 51: $43-46$

4 Reichert H, Eng PJ, Dosch H, et al. Thermodynamics of surface segregation profiles at $\mathrm{Cu}_{3} \mathrm{Au}(001)$ resolved by X-ray scattering. Phys Rev Lett, 1995, 74: 2006-2009

5 Vasiliev MA. Surface effects of ordering in binary alloys. J Phys DAppl Phys, 1997, 30: 3037-3070

6 Han BC, van der Ven A, Ceder G, et al. Surface segregation and ordering of alloy surfaces in the presence of adsorbates. Phys Rev B, 2005, 72: 205409

7 Zafeiratos S, Piccinin S, Teschner D. Alloys in catalysis: Phase separation and surface segregation phenomena in response to the reactive environment. Catal Sci Technol, 2012, 2: 1787-1801

8 Toshima N, Yonezawa T. Bimetallic nanoparticles: Novel materials for chemical and physical applications. New J Chem, 1998, 22: 1179-1201

9 Cui C, Gan L, Heggen M, et al. Compositional segregation in shaped Pt alloy nanoparticles and their structural behaviour during electrocatalysis. Nat Mater, 2013, 12: 765-771

10 Zhan W, Wang J, Wang $\mathrm{H}$, et al. Crystal structural effect of $\mathrm{AuCu}$ alloy nanoparticles on catalytic $\mathrm{CO}$ oxidation. J Am Chem Soc, 2017, 139: 8846-8854

11 Yang F, Zhao H, Wang X, et al. Atomic scale stability of tungstencobalt intermetallic nanocrystals in reactive environment at high 
temperature. J Am Chem Soc, 2019, 141: 5871-5879

$12 \mathrm{Wu} \mathrm{CH}$, Liu C, Su D, et al. Bimetallic synergy in cobalt-palladium nanocatalysts for CO oxidation. Nat Catal, 2019, 2: 78-85

13 Gocyla M, Kuehl S, Shviro M, et al. Shape stability of octahedral PtNi nanocatalysts for electrochemical oxygen reduction reaction studied by in situ transmission electron microscopy. ACS Nano, 2018, 12: 5306-5311

14 Watson DJ, Attard GA. Surface segregation and reconstructive behaviour of the (100) and (110) surfaces of platinum-palladium bulk alloy single crystals: A voltammetric and LEED/AES study. Surf Sci, 2002, 515: 87-93

15 Polak M, Rubinovich L. The interplay of surface segregation and atomic order in alloys. Surf Sci Rep, 2000, 38: 127-194

16 Dai S, You Y, Zhang S, et al. In situ atomic-scale observation of oxygen-driven core-shell formation in $\mathrm{Pt}_{3} \mathrm{Co}$ nanoparticles. Nat Commun, 2017, 8: 204

17 Shen X, Zhang C, Zhang S, et al. Deconvolution of octahedral $\mathrm{Pt}_{3} \mathrm{Ni}$ nanoparticle growth pathway from in situ characterizations. Nat Commun, 2018, 9: 4485

18 Zhang S, Chen C, Cargnello M, et al. Dynamic structural evolution of supported palladium-ceria core-shell catalysts revealed by in situ electron microscopy. Nat Commun, 2015, 6: 7778

19 Hansen TW, Wagner JB. Controlled Atmosphere Transmission Electron Microscopy. Cham: Springer, 2016

20 Bugnet M, Overbury SH, Wu ZL, et al. Direct visualization and control of atomic mobility at $\{100\}$ surfaces of ceria in the environmental transmission electron microscope. Nano Lett, 2017, 17: 7652-7658

21 Zhang S, Plessow PN, Willis JJ, et al. Dynamical observation and detailed description of catalysts under strong metal-support interaction. Nano Lett, 2016, 16: 4528-4534

22 Tao FF, Crozier PA. Atomic-scale observations of catalyst structures under reaction conditions and during catalysis. Chem Rev, 2016, 116: 3487-3539

23 Zou L, Saidi WA, Lei Y, et al. Segregation induced order-disorder transition in $\mathrm{Cu}(\mathrm{Au})$ surface alloys. Acta Mater, 2018, 154: 220-227

24 Zou L, Yang C, Lei Y, et al. Dislocation nucleation facilitated by atomic segregation. Nat Mater, 2017, 17: 56-63

25 Gómez-Rodríguez A, Beltrán-Del-Río LM, Herrera-Becerra R. SimulaTEM: Multislice simulations for general objects. Ultramicroscopy, 2010, 110: 95-104

26 Luo L, Chen S, Xu Q, et al. Dynamic atom clusters on $\mathrm{AuCu}$ nanoparticle surface during CO oxidation. J Am Chem Soc, 2020, 142: 4022-4027

27 He Y, Liu JC, Luo L, et al. Size-dependent dynamic structures of supported gold nanoparticles in CO oxidation reaction condition. Proc Natl Acad Sci USA, 2018, 115: 7700-7705

28 Wang YG, Mei D, Glezakou VA, et al. Dynamic formation of single-atom catalytic active sites on ceria-supported gold nanoparticles. Nat Commun, 2015, 6: 6511

29 Müller P, Kern R. Equilibrium nano-shape changes induced by epitaxial stress (generalised Wulf-Kaishew theorem). Surf Sci, 2000, 457: 229-253

30 Song M, Zhou G, Lu N, et al. Oriented attachment induces fivefold twins by forming and decomposing high-energy grain boundaries. Science, 2020, 367: 40-45

Acknowledgements This work was supported by the National Natural Science Foundation of China (21873069 and 11504162). The authors would like to thank the Collaborative Innovation Center of Sustainable
Energy Materials in Guangxi University for the use of the aberrationcorrected HRTEM facility.

Author contributions Luo L and Zhang L conceived this study and designed the experiments; Zhang $\mathrm{L}$ and Dong $\mathrm{Z}$ carried out the sample preparation and series of TEM experiments; Zhang L analyzed all the data and wrote the draft of the paper with the help of all the authors. All authors discussed the results and commented on the manuscript.

Conflict of interest The authors declare that they have no conflict of interest.

Supplementary information Experimental details, supporting data and movies are available in the online version of the paper.

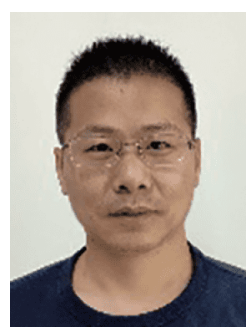

Lifeng Zhang received his $\mathrm{PhD}$ degree from the Institute of Metal Research, Chinese Academy of Sciences. Currently, he is a lecturer at the Institute of Molecular Plus at Tianjin University in China. His current research interest focuses on the advanced (S)TEM studies of microstructure, interface and complicated defects in materials to reveal their related processing, structure and properties.

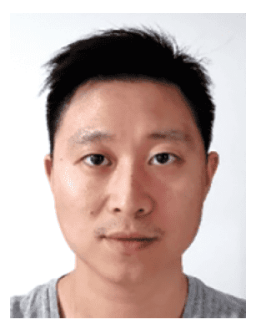

Langli Luo received his $\mathrm{PhD}$ degree in materials science and engineering from the State University of New York at Binghamton. He was a postdoctoral fellow at Northwestern University and Pacific Northwest National Laboratory in USA. Currently, he is a professor at the Institute of Molecular Plus, Tianjin University in China. His current research focuses on in situ TEM studies of chemical processes and energy-related materials.

\section{气体吸附诱导 $\mathrm{Cu}(\mathrm{Au})$ 合金中 $\mathrm{Au}$ 原子的偏聚和富集}

\author{
张利峰 ${ }^{1}$, 董泽建 ${ }^{1}$, 王双宝 ${ }^{2}$, 罗浪里 ${ }^{*}$
}

摘要 合金中的表面成分和相偏析会改变其功能, 尤其是在表面结 构和化学起着至关重要作用的应用中. 例如, 合金催化剂的表面状 态显著影响其在异相催化和电化学过程中的催化性能. 表面成分 偏析被认为是由表面能的差异驱动, 以减少合金体系的总自由能. 然而, 目前合金中成分偏析的原子尺度进程还尚不清楚, 尤其是对 于气体分子诱导的成分偏析, 在该过程中可能同时发生结构和化 学重排. 本文通过像差校正环境TEM从原子尺度研究了固溶态 $\mathrm{Cu}(\mathrm{Au})$ 合金在 $\mathrm{CO}$ 气氛下的表面偏析行为. $\mathrm{CO}$ 气氛能够诱导 $\mathrm{Cu}$ $(\mathrm{Au})$ 合金表面形成有序结构, 在很大程度上改变合金的表面化学 性质. 高温条件下, $\mathrm{CO}$ 气氛会进一步促进 $\mathrm{Au}$ 原子通过特定的“原子 通道”进行扩散，在合金表面偏聚和富集. 富集形成的 $\mathrm{Au}$ 纳米颗粒 与合金表面在形貌和结构方面发生了丰富的动力学交互作用. 这 其中 $\mathrm{CO}$ 气体吸附也起着重要的作用. 这些原子尺度的研究结果为 双金属合金的表面偏析和去合金化机理提供了直接证据, 并突出 了气体吸附物在这些表面行为中的作用. 